\title{
Structure of Acoustic Field in Infrared Tellurium Based $\boldsymbol{X}-\boldsymbol{Y}$ Deflector
}

\author{
A.S. TRUSHIN ${ }^{a, *}$ AND V.B. VOLOSHINOV ${ }^{b}$ \\ ${ }^{a}$ Lebedev Physical Institute RAS, 119991, 53 Leninskij Prospekt, Moscow, Russia \\ ${ }^{b}$ Lomonosov Moscow State University, 119991, GSP-1, Leninskie Gory, Moscow, Russia
}

\begin{abstract}
Theoretical investigation of acoustic field in a tellurium two-coordinate deflector was carried out. We used angular spectrum method supported by consideration to boundary conditions in approximation of a given displacement to calculate spatial distribution of the acoustic field intensity. Volumetric illustration is presented for the acoustic beam launched from $Y Z$ face of the crystal. Cross-sections of the acoustic field generated from $X Z$ face are also presented in the work. It is shown that the strong anisotropy of acoustic properties in the Te crystal leads to principally different structure of the two fields excited from the $X Z$ and $Y Z$ crystal faces. The acoustic field excited from the $X Z$ face may be considered as a simple beam with a relatively high divergence. On the other hand, the field generated from the $Y Z$ face looks like superposition of two beams with relatively low divergence. Ratio of intensities of the two beams generated by longitudinal piezotransducers is equal to the ratio predicted by analysis based by plane-wave theory.
\end{abstract}

DOI: $10.12693 /$ APhysPolA.127.55

PACS: $02.30 . \mathrm{Nw}, 02.60 . \mathrm{Cb}, 02.70 . \mathrm{Hm}, 43.20 . \mathrm{Gp}, 46.40 . \mathrm{Cd}, 46.15 .-\mathrm{x}$

\section{Introduction}

Development of devices operating not only in the visible light but also in the infrared and ultraviolet spectral ranges is the task of utmost importance in acousto-optics. The major fundamental problem of acousto-optics (AO) in the infrared spectral range is a decrease of diffraction efficiency with an increase of electromagnetic wavelength. Therefore, in order to develop $\mathrm{AO}$ devices operating with low electric power consumption, it is necessary to use a material with a high magnitude of the acousto-optic figure of merit $M_{2}$. One of the prominent materials of infrared optics, in general, and acousto-optics, in particular is the crystalline tellurium. It is a well-established fact that this material demonstrates very high magnitude of the coefficient $M_{2}$ for some geometries of AO interaction [1]. A few papers have been devoted to theoretical and experimental investigations of a two-coordinate tellurium deflector $[2-4]$. The carried out studies revealed discrepancies between theoretical predictions and the results of experimental investigations [2]. One of the possible reasons for these discrepancies originates from simplicity of the considered acoustic-field model. Traditionally calculation of direction of wave propagation and estimations of effective length of acousto-optic interaction were carried out in the assumption of the acoustic field consisting only of a single harmonic plane wave with an angular spectrum in a form of delta-function. Finite width of angular spectra of acoustic beams and anisotropy of elastic properties in tellurium crystal must be also taken into account. In the present paper, our interest was concentrated on analysis of acoustic field generation in tellurium two-coordinate deflector.

*corresponding author; e-mail: a.trushin@physics.msu.ru

\section{Calculation method}

We calculated distribution of acoustic field parameters in space using a modified angular spectrum method generalized over an anisotropic medium [5]. Three tensor fields must be examined in the analysis: mechanical displacement field, strain field and stress field. We found that it is possible to consider components of these fields $u_{i}(x, y, z), S_{i j}(x, y, z)$, and $T_{i j}(x, y, z)$ as independent scalar fields. It is convenient to denote formally $u_{i}, S_{i j}$ and $T_{i j}(x, y, z)$ as components of the vector $Q^{r}$, where $r$ is an index which selects a required component. Therefore, computation of the acoustic field parameters can be conducted in accordance with the following expression:

$$
\begin{aligned}
& Q^{r}(x, y, z)=\iint_{\infty} \mathrm{d} k_{x} \mathrm{~d} k_{y} A\left(k_{x}, k_{y}\right) \sum_{p=1}^{3} B_{p}\left(k_{x}, k_{y}\right) \\
& \quad \times Q_{p}^{r}\left(k_{x}, k_{y}\right) \exp \left(\mathrm{i}\left(\omega t-\boldsymbol{k}_{p} \boldsymbol{r}\right)\right),
\end{aligned}
$$

where $Q^{r}(x, y, z)$ is the spatial distribution of the displacement vector, as well as the stress and strain tensors, $A\left(k_{x}, k_{y}\right)$ is its two-dimensional Fourier transform of the displacement profile $u(x, y)$, to be mentioned below in Eq. (2), $\boldsymbol{k}$ is the acoustic wave vector, $\boldsymbol{r}=\{x, y, z\}$ is the radius vector, $\omega$ is the circular frequency, $p$ is the index representing a group of waves with equal projections of their wave vector on the $X Y$ plane, $B_{p}$ are the weights of each plane-wave component in such a group, $Q_{p}^{r}$ are the components of a polarization vector, as well as the stress and strain tensors for a single elementary wave of a normalized amplitude and known projection of the wave vector $k_{x}$ and $k_{y}$.

The values $B_{p}$ can be found from the boundary conditions. Two types of the boundary conditions are known: a "given force" and a "given displacement". We chose considering the case of a "given displacement" type. For the sake of simplicity, we expressed the displacement of the crystal surface in the form 


$$
\boldsymbol{u}(x, y)=\boldsymbol{u}_{0} u(x, y),
$$

where $\boldsymbol{u}_{0}$ is the unit displacement vector and $u(x, y)$ is the amplitude of the displacement on the crystal surface. Rigorous calculation of $u(x, y)$ is a complex task, so we assumed that inside a piezo-transducer the displacement $u(x, y)=1$ while outside we have $u(x, y)=0$. We use a longitudinal piezo-transducer, therefore in the laboratory coordinate system $\boldsymbol{u}_{0}=\{0,0,1\}$. For the chosen type of the boundary conditions, one can write the following equation for the $B_{p}$ terms:

$$
\boldsymbol{u}_{0}=\sum_{p=1}^{3} B_{p}\left(k_{x}, k_{y}\right) \boldsymbol{q}_{p}\left(k_{x}, k_{y}\right),
$$

where $\boldsymbol{q}_{p}\left(k_{x}, k_{y}\right)$ is the polarization of a plane wave with the index $p$ and projection of the wave vector $\left(k_{x}, k_{y}\right)$. Let us note that $\boldsymbol{q}_{p}$ is a function of $\left(k_{x}, k_{y}\right)$. However, due to linear mutual independence of $\boldsymbol{q}_{1}, \boldsymbol{q}_{2}$ and $\boldsymbol{q}_{3}$ it is possible to determine $B_{p}\left(k_{x}, k_{y}\right)$ in order to satisfy $(3)$.

A calculation procedure that implements this method requires to have steps: (1) calculation of $A\left(k_{x}, k_{y}\right)$ using fast Fourier transform; (2) selection of an elementary solution based on the sign of the third component of group velocity, for homogeneous waves and based on sign of Im $k_{z}$, for inhomogeneous waves; (3) calculation of polarization $\boldsymbol{q}$, strain $S$ and stress $T$ for each of the three selected waves; (4) calculation of the weight $B_{p}\left(k_{x}, k_{y}\right)$; (5) calculation of the displacement $\boldsymbol{u}(x, y, z)$, the strain $S(x, y, z)$ and the stress $T(x, y, z)$ using Eq. (1) and fast Fourier transform.

\section{Acousto-optic cell geometry}

Configuration of the tellurium deflector is shown in Fig. 1. We use two longitudinal piezo-transducers to launch ultrasound beams in the tellurium crystal shaped as a cube with the size $a=1.3 \mathrm{~cm}$. Edges of the cube are collinear with the basic axes of the crystal. The $Y Z$ transducer is perpendicular to the $X$-axis, and has dimensions $\left(l, h_{x}\right)=(7.5,3) \mathrm{mm}$. In order to increase density of the acoustic field energy to obtain diffraction efficiency close to $100 \%$, we decrease height $h_{x}$. Therefore, shape of $Y Z$-transducer is not square. The $X Z$ transducer is perpendicular to the $Y$-axis and has the dimensions $\left(l, h_{y}\right)=(7.5,6) \mathrm{mm}$. Direction of light propagation in this device is close to the $Z$-axis, the frequency $f=20 \mathrm{MHz}$ was typical for the examined device usually operating in the frequency range from 10 to $35 \mathrm{MHz}$. Variation of the frequency in this range really affects the divergence of the acoustic beam launched only by the $Y Z$-transducer because slowness surfaces demonstrate big curvature near the $X$-axis [1]. The structure of the field launched by the $X Z$ transducer is frequencyindependent for the mentioned range because the slowness surfaces of tellurium crystals are almost flat near the $Y$-axis.

The acoustic intensity in region shown in Fig. 2a is higher than 0.5 of the maximal value. It is obvious that the edge of the beam is not collinear with the edge of the piezotransducer and the angle between them

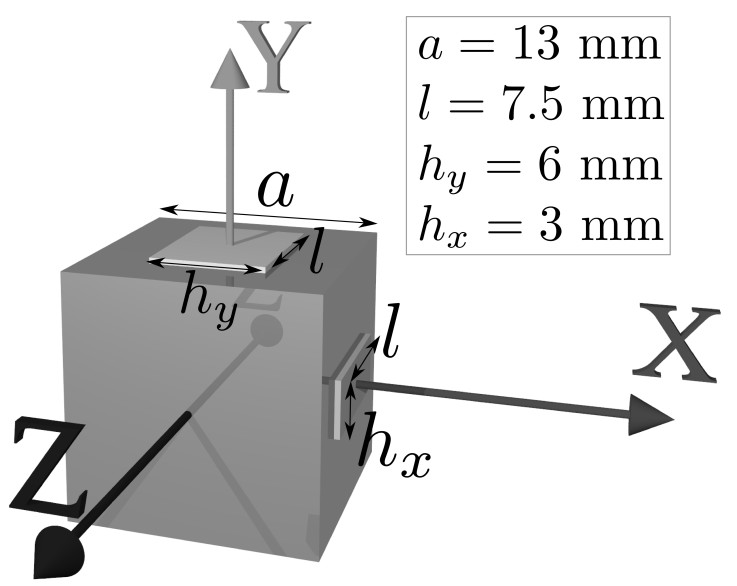

Fig. 1. Two-coordinate tellurium acousto-optic deflector cell applying light propagating along the $z$-axis.
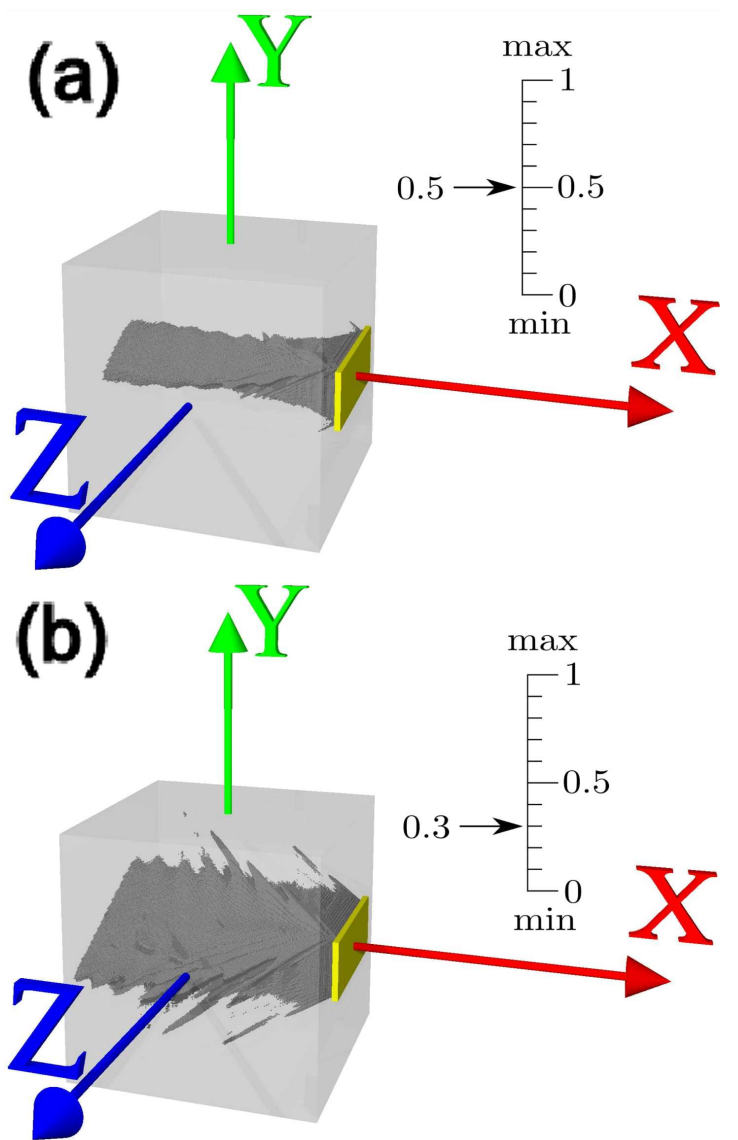

Fig. 2. Volumetric representation of the acoustic field excited by the $Y Z$-transducer with different levels of visualization threshold $I_{\mathrm{cr}}$ : (a) $I_{\mathrm{cr}}=0.5$, (b) $I_{\mathrm{cr}}=0.3$.

is approximately equal to $30^{\circ}$. However, the edge of the beam in Fig. $2 b$ has more obtuse angle, which is close to $45^{\circ}$. Therefore, beam is "twisted". It is impossible to obtain this result from $2 \mathrm{D}$ analysis.

Figures 3 and 4 show distribution of acoustical field intensity. The black color corresponds to the zero field intensity and the white to the maximal field intensity. 
Figure 3 shows the same field as Fig. 2. One can see that distribution of the field intensity is non-uniform and demonstrates behavior that is very unlike to diffraction patterns in isotropic media.

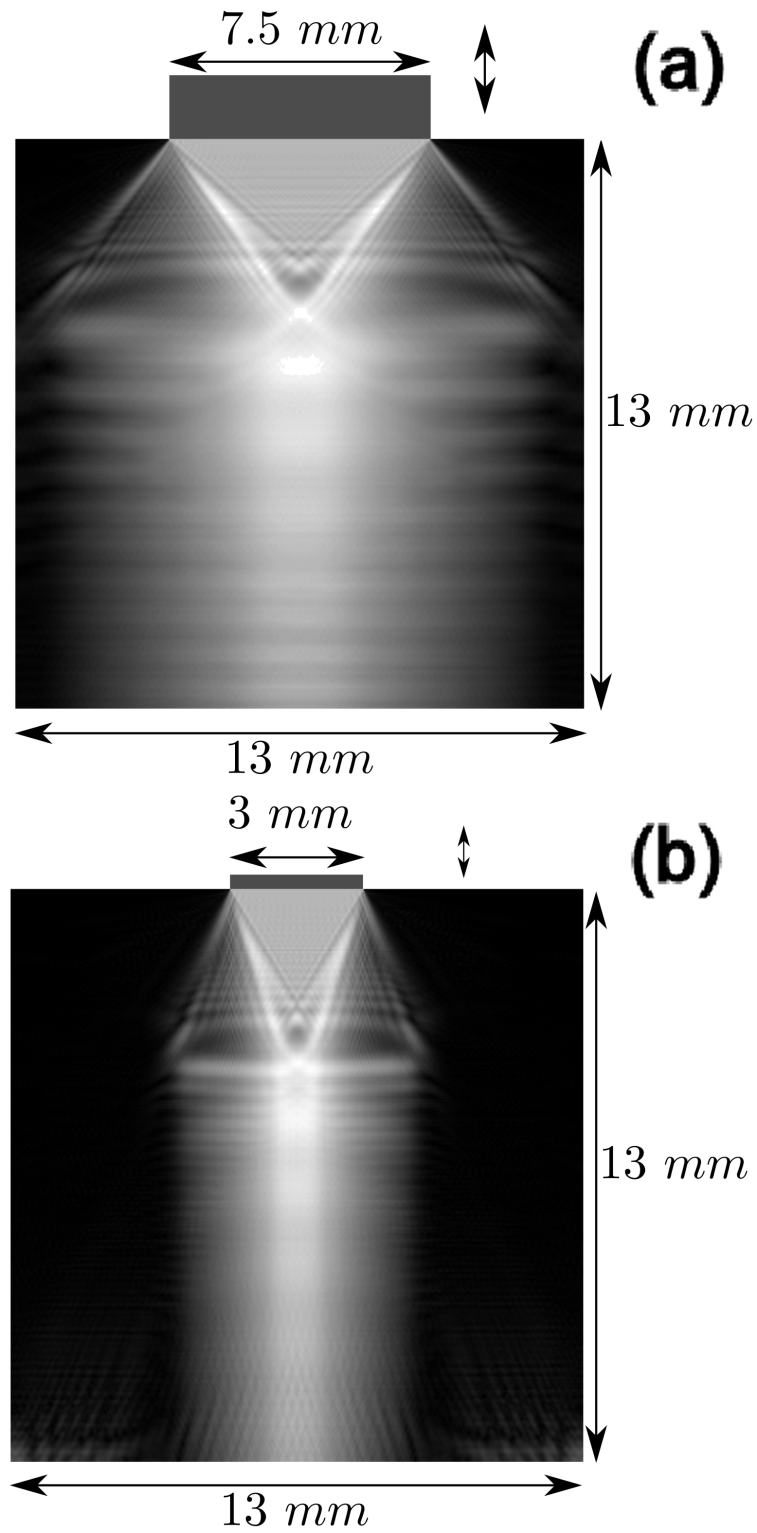

Fig. 3. Structure of the acoustic field excited by the $Y Z$-transducer: (a) cross-section by the $\mathrm{ZX}$ plane, (b) cross-section by the $Y X$ plane.

Figure 4 shows the acoustic field structure for the $X Z$ transducer. This sound field consists of two beams with low divergence and relatively simple intrinsic structure. One beam corresponds to quasi-longitudinal wave with phase velocity $2900 \mathrm{~m} / \mathrm{s}$ and the other corresponds to the quasi-shear wave with phase velocity $1900 \mathrm{~m} / \mathrm{s}$. The angles between the phase and group velocities for these beams are in a good agreement with the results of planewave analysis known from literature [2]. Intensities of these beams are roughly equal to each other that agrees with the results of the plane-wave analysis.

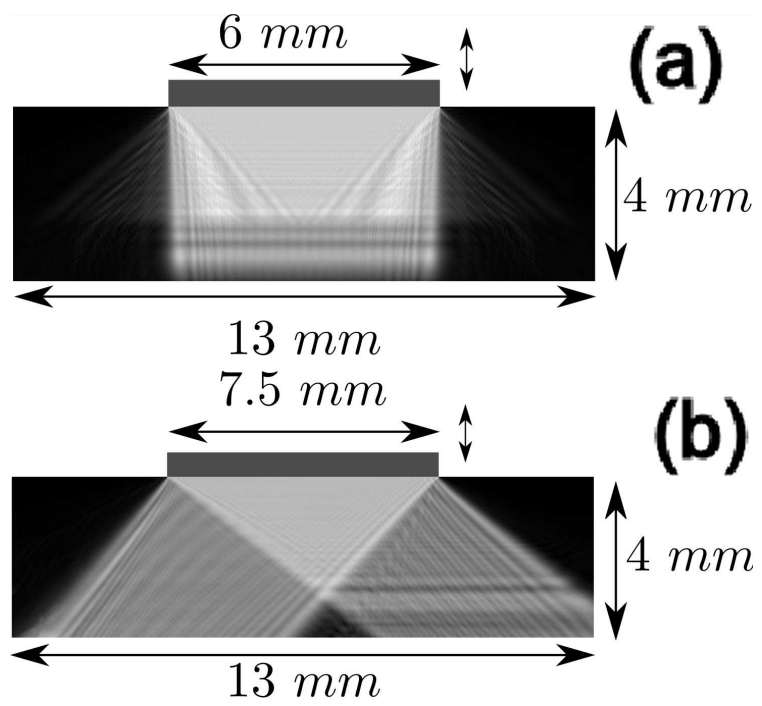

Fig. 4. Cross-sections of the acoustic field excited by the $X Z$-transducer: (a) cross-section by the $Y X$ plane, (b) cross section by the $Y Z$ plane.

\section{Conclusions}

Analysis of structure of the acoustic field in the tellurium AO cell carried out by angular spectrum method shows that longitudinal piezo-transducers attached to the $X Y$ - and $X Z$-planes excite different types of acoustic waves. The field originated from the $X Y$-transducer consists of two low-divergent beams with almost equal intensities, while the acoustic beam coming from the $Y Z$-transducer has a relatively strong divergence and a complicated intrinsic structure. The angles between the phase and group velocities of these beams are close to those obtained by plane-wave analysis. However, some features of the acoustic field in this cell such as its "twisted" structure can be considered only by application of three-dimensional representation of the beam.

\section{Acknowledgments}

The "Russian Science Foundation" under the project number 141200380 supports this research.

\section{References}

[1] V.B. Voloshinov, V.I. Balakshy, L.A. Kulakova, N. Gupta, J. Opt. A Pure Appl. Opt. 10, 095002 (2008).

[2] V.B. Voloshinov, N. Gupta, G.A. Knyazev, N.V. Polikarpova, J. Opt. 13, 015706 (2011).

[3] R.W. Dixon, A.N. Chester, Appl. Phys. Lett. 9, 5 (1966).

[4] A.M. Diakonov, Y.V. Ilisabsky, I.I. Farbstein, Sov. Tech. Phys. Lett. 3, 567 (1977) (in Russian).

[5] D.J. Vezzetti, J. Acoust. Soc. Am. 78, 1103 (1985). 